\section{Volume 66}

Issue 66, I (pag. 3- 96): I4-10-1979

Issue 66, 2 (pag. 99-192): I-10-1 979

Issue 66, 3 (pag. 195-208): 15-10-1 979

\section{INDEX AUCTORUM}

Armitage, P. D., 245

Asif, M., 49

Atchison, G. J., 105

Bachman, R. A., 45

Balirwa, J. S., 65

Bluzat, R., 25

Casterlin, M. E., 45, 141, 223

Crayton, W. M., 8 I

Dumont, H. J., 259

Duursma, E. K., 279

Fernando, C. H., I I 3

Frandsen, F., I 7

Fuchs, I. R., 209

Gophen, M., 4I, 99

Graneli, W., I 49

Hannan, H. H., 209

Jurkovic, A. A., 33
Kalk, M., 7

Kogelschatz, J. E., 195

Lam, C. W. Y., 237

Laureys, P., 259

LaVelle, J. W., 227

Learner, M. A., I 35

Litsky, W., 249

Lubbock, R., 3

Madsen, H., I7, 18 I

Magdyck, W. P., I6I

Mayes, R. A., 105

McIntosh, A. W., 105

Michaud, M. T., 105

Mishra, A. P., 129

Moore, J. W., 73

Nakajima, T., 57
Kwiatkowski, R. E., 33
Nelson, D. W., 105

Pennak, R. W., 227

Pensaert, J., 259

Rao, S. S., 33

Reynolds, W. W., 45, I4I, 145, 223

Rho, J., 249

Robothan, P. W. J., I 77

Saxena, R., 275

Seugé, J., 25

Singh, B. R., I 29

Sommerfield, M. R., 8 I

Sorokin, Y, I., I 95

Swar, D. B., I 13

Tyagi, A. T., 275

Whitenberg, D. C., 209

Zandee, D. I., 279 